\title{
Bicrossed Products, Matched Pair Deformations and the Factorization Index for Lie Algebras
}

\author{
Ana-Loredana AGORE ${ }^{\dagger \ddagger}$ and Gigel MILITARU $\S$ \\ $\dagger$ Faculty of Engineering, Vrije Universiteit Brussel, Pleinlaan 2, B-1050 Brussels, Belgium \\ E-mail: ana.agore@vub.ac.be,ana.agore@gmail.com \\ URL: http://homepages.vub.ac.be/ aagore/ \\ $\ddagger$ Department of Applied Mathematics, Bucharest University of Economic Studies, \\ Piata Romana 6, RO-010374 Bucharest 1, Romania \\ $\S$ Faculty of Mathematics and Computer Science, University of Bucharest, \\ Str. Academiei 14, RO-010014 Bucharest 1, Romania \\ E-mail: gigel.militaru@fmi.unibuc.ro,gigel.militaru@gmail.com \\ URL: http://fmi.unibuc.ro/ro/departamente/matematica/militaru_gigel/
}

Received January 20, 2014, in final form June 10, 2014; Published online June 16, 2014

http://dx.doi.org/10.3842/SIGMA.2014.065

\begin{abstract}
For a perfect Lie algebra $\mathfrak{h}$ we classify all Lie algebras containing $\mathfrak{h}$ as a subalgebra of codimension 1. The automorphism groups of such Lie algebras are fully determined as subgroups of the semidirect product $\mathfrak{h} \ltimes\left(k^{*} \times \operatorname{Aut}_{\text {Lie }}(\mathfrak{h})\right)$. In the non-perfect case the classification of these Lie algebras is a difficult task. Let $\mathfrak{l}(2 n+1, k)$ be the Lie algebra with the bracket $\left[E_{i}, G\right]=E_{i},\left[G, F_{i}\right]=F_{i}$, for all $i=1, \ldots, n$. We explicitly describe all Lie algebras containing $\mathfrak{l}(2 n+1, k)$ as a subalgebra of codimension 1 by computing all possible bicrossed products $k \bowtie \mathfrak{l}(2 n+1, k)$. They are parameterized by a set of matrices $\mathrm{M}_{n}(k)^{4} \times k^{2 n+2}$ which are explicitly determined. Several matched pair deformations of $\mathfrak{l}(2 n+1, k)$ are described in order to compute the factorization index of some extensions of the type $k \subset k \bowtie \mathfrak{l}(2 n+1, k)$. We provide an example of such extension having an infinite factorization index.
\end{abstract}

Key words: matched pairs of Lie algebras; bicrossed products; factorization index

2010 Mathematics Subject Classification: 17B05; 17B55; 17B56

\section{Introduction}

The theory of Lie algebras is among the most developed fields in algebra due to his broad applicability in differential geometry, theoretical physics, quantum field theory, classical or quantum mechanics and others. Besides the purely algebraic interest in this problem, the classification of Lie algebras of a given dimension is a central theme of study in modern group analysis of differential equations - for further explanations and an historical background see [21]. The Levi-Malcev theorem reduces the classification of all finite-dimensional Lie algebras over a field of characteristic zero to the following three subsequent problems: (1) the classification of all semi-simple Lie algebras (solved by Cartan); (2) the classification of all solvable Lie algebras (which is known up to dimension 6 [8]) and (3) the classification of all Lie algebras that are direct sums of semi-simple Lie algebras and solvable Lie algebras.

Surprisingly, among these three problems, the last one is the least studied and the most difficult. Only in 1990 Majid [16, Theorem 4.1] and independently Lu and Weinstein [15, Theorem 3.9] introduced the concept of a matched pair between two Lie algebras $\mathfrak{g}$ and $\mathfrak{h}$. To any matched pair of Lie algebras we can associate a new Lie algebra $\mathfrak{g} \bowtie \mathfrak{h}$ called the 
bicrossed product (also called double Lie algebra in [15, Definition 3.3], double cross sum in [17, Proposition 8.3.2] or knit product in [19]). In light of this new concept, problem (3) can be equivalently restated as follows: for a given (semi-simple) Lie algebra $\mathfrak{g}$ and a given (solvable) Lie algebra $\mathfrak{h}$, describe the set of all possible matched pairs $(\mathfrak{g}, \mathfrak{h}, \triangleleft, \triangleright)$ and classify up to an isomorphism all associated bicrossed products $\mathfrak{g} \bowtie \mathfrak{h}$. Leaving aside the semi-simple/solvable case this is just the factorization problem for Lie algebras - we refer to [1] for more details and additional references on the factorization problem at the level of groups, Hopf algebras, etc.

The present paper continues our recent work [3,4] related to the above question (3), in its general form, namely the factorization problem and its converse, called the classifying complement problem, which consist of the following question: let $\mathfrak{g} \subset \mathfrak{L}$ be a given Lie subalgebra of $\mathfrak{L}$. If a complement of $\mathfrak{g}$ in $\mathfrak{L}$ exists (that is a Lie subalgebra $\mathfrak{h}$ such that $\mathfrak{L}=\mathfrak{g}+\mathfrak{h}$ and $\mathfrak{g} \cap \mathfrak{h}=\{0\}$ ), describe explicitly, classify all complements and compute the cardinal of the isomorphism classes of all complements (which will be called the factorization index $[\mathfrak{L}: \mathfrak{g}]^{f}$ of $\mathfrak{g}$ in $\mathfrak{L}$ ). Our starting point is [4, Proposition 4.4] which describes all Lie algebras $\mathfrak{L}$ that contain a given Lie algebra $\mathfrak{h}$ as a subalgebra of codimension 1 over an arbitrary field $k$ : the set of all such Lie algebras $L$ is parameterized by the space TwDer $(\mathfrak{h})$ of twisted derivations of $\mathfrak{h}$. The pioneer work on this subject was performed by K.H. Hofmann: [12, Theorem I] describes the structure of $n$-dimensional real Lie algebras containing a given subalgebra of dimension $n-1$. Equivalently, this proves that the set of all matched pairs of Lie algebras $\left(k_{0}, \mathfrak{h}, \triangleleft, \triangleright\right)$ (by $k_{0}$ we will denote the Abelian Lie algebra of dimension 1) and the space $\operatorname{TwDer}(\mathfrak{h})$ of all twisted derivations of $\mathfrak{h}$ are in one-to-one correspondence; moreover, any Lie algebra $\mathfrak{L}$ containing $\mathfrak{h}$ as a subalgebra of codimension 1 is isomorphic to a bicrossed product $k_{0} \bowtie \mathfrak{h}=\mathfrak{h}_{(\lambda, \Delta)}$, for some $(\lambda, \Delta) \in \operatorname{TwDer}(\mathfrak{h})$. The classification up to an isomorphism of all bicrossed products $\mathfrak{h}_{(\lambda, \Delta)}$ is given in the case when $\mathfrak{h}$ is perfect. As an application of our approach, the group $\operatorname{Aut}_{L i e}\left(\mathfrak{h}_{(\lambda, \Delta)}\right)$ of all automorphisms of such Lie algebras is fully described in Corollary 3.3: it appears as a subgroup of a certain semidirect product $\mathfrak{h} \ltimes\left(k^{*} \times \operatorname{Aut}_{\text {Lie }}(\mathfrak{h})\right)$ of groups. At this point we mention that the classification of automorphisms groups of all indecomposable real Lie algebras of dimension up to five was obtained recently in [11] where the importance of this subject in mathematical physics is highlighted. For the special case of sympathetic Lie algebras $\mathfrak{h}$, Corollary 3.5 proves that, up to an isomorphism, there exists only one Lie algebra that contains $\mathfrak{h}$ as a Lie subalgebra of codimension one, namely the direct product $k_{0} \times \mathfrak{h}$ and $\operatorname{Aut}_{\text {Lie }}\left(k_{0} \times \mathfrak{h}\right) \cong k^{*} \times \operatorname{Aut}_{\text {Lie }}(\mathfrak{h})$. Now, $k_{0}$ is a subalgebra of $k_{0} \bowtie \mathfrak{h}=\mathfrak{h}_{(\lambda, \Delta)}$ having $\mathfrak{h}$ as a complement: for a 5-dimensional perfect Lie algebra all complements of $k_{0}$ in $\mathfrak{h}_{(\lambda, \Delta)}$ are described in Example 3.7 as matched pair deformations of $\mathfrak{h}$. Section 4 treats the same problem for a given $(2 n+1)$-dimensional non-perfect Lie algebra $\mathfrak{h}:=\mathfrak{l}(2 n+1, k)$. Theorem 4.2 describes explicitly all Lie algebras containing $\mathfrak{l}(2 n+1, k)$ as a subalgebra of codimension 1 . They are parameterized by a set $\mathcal{T}(n)$ of matrices $\left(A, B, C, D, \lambda_{0}, \delta\right) \in \mathrm{M}_{n}(k)^{4} \times k \times k^{2 n+1}$ : there are four such families of Lie algebras if the characteristic of $k$ is $\neq 2$ and two families in characteristic 2. All complements of $k_{0}$ in two such bicrossed products $k_{0} \bowtie \mathfrak{l}(2 n+1, k)$ are described by computing all matched pair deformations of the Lie algebra $\mathfrak{l}(2 n+1, k)$ in Propositions 4.4 and 4.8. In particular, in Example 4.6 we construct an example where the factorization index of $k_{0}$ in the 4-dimensional Lie algebra $\mathfrak{m}(4, k)$ is infinite: that is $k_{0}$ has an infinite family of non-isomorphic complements in $\mathfrak{m}(4, k)$. To conclude, there are three reasons for which we considered the Lie algebra $\mathfrak{l}(2 n+1, k)$ in Section 4 : on the one hand it provided us with an example of a finite-dimensional Lie algebra extension $\mathfrak{g} \subset \mathfrak{L}$ such that $\mathfrak{g}$ has infinitely many non-isomorphic complements as a Lie subalgebra in $\mathfrak{L}$. On the other hand, the Lie algebra $\mathfrak{l}(2 n+1, k)$ serves for constructing two counterexamples in Remark 4.10 which show that some properties of Lie algebras are not preserves by the matched pair deformation. Finally, having [2, Corollary 3.2] as a source of inspiration we believe that any $(2 n+1)$-dimensional Lie algebra is isomorphic to an $r$-deformation of $\mathfrak{l}(2 n+1, k)$ associated to a given matched pair: a more general open question is stated at the end of the paper. 


\section{Preliminaries}

All vector spaces, Lie algebras, linear or bilinear maps are over an arbitrary field $k$. The Abelian Lie algebra of dimension $n$ will be denoted by $k_{0}^{n}$. For two given Lie algebras $\mathfrak{g}$ and $\mathfrak{h}$ we denote by $\operatorname{Aut}_{\text {Lie }}(\mathfrak{g})$ the group of automorphisms of $\mathfrak{g}$ and by $\operatorname{Hom}_{\text {Lie }}(\mathfrak{g}, \mathfrak{h})$ the space of all Lie algebra maps between $\mathfrak{g}$ and $\mathfrak{h}$. A Lie algebra $\mathfrak{L}$ factorizes through $\mathfrak{g}$ and $\mathfrak{h}$ if $\mathfrak{g}$ and $\mathfrak{h}$ are Lie subalgebras of $\mathfrak{L}$ such that $\mathfrak{L}=\mathfrak{g}+\mathfrak{h}$ and $\mathfrak{g} \cap \mathfrak{h}=\{0\}$. In this case $\mathfrak{h}$ is called a complement of $\mathfrak{g}$ in $\mathfrak{L}$; if $\mathfrak{g}$ is an ideal of $\mathfrak{L}$, then a complement $\mathfrak{h}$, if it exists, is unique being isomorphic to the quotient Lie algebra $\mathfrak{L} / \mathfrak{g}$. In general, if $\mathfrak{g}$ is only a subalgebra of $\mathfrak{L}$, then we are very far from having unique complements; for a given extension $\mathfrak{g} \subset \mathfrak{L}$ of Lie algebras, the number of types of isomorphisms of all complements of $\mathfrak{g}$ in $\mathfrak{L}$ is called the factorization index of $\mathfrak{g}$ in $\mathfrak{L}$ and is denoted by $[\mathfrak{L}: \mathfrak{g}]^{f}$ a theoretical formula for computing $[\mathfrak{L}: \mathfrak{g}]^{f}$ is given in $[3$, Theorem 4.5]. For basic concepts and unexplained notions on Lie algebras we refer to $[9,13]$.

A matched pair of Lie algebras $[15,17]$ is a system $(\mathfrak{g}, \mathfrak{h}, \triangleleft, \triangleright)$ consisting of two Lie algebras $\mathfrak{g}$ and $\mathfrak{h}$ and two bilinear maps $\triangleright: \mathfrak{h} \times \mathfrak{g} \rightarrow \mathfrak{g}, \triangleleft: \mathfrak{h} \times \mathfrak{g} \rightarrow \mathfrak{h}$ such that $(\mathfrak{g}, \triangleright)$ is a left $\mathfrak{h}$-module, $(\mathfrak{h}, \triangleleft)$ is a right $\mathfrak{g}$-module and the following compatibilities hold for all $g, h \in \mathfrak{g}$ and $x, y \in \mathfrak{h}$

$$
\begin{aligned}
& x \triangleright[g, h]=[x \triangleright g, h]+[g, x \triangleright h]+(x \triangleleft g) \triangleright h-(x \triangleleft h) \triangleright g, \\
& {[x, y] \triangleleft g=[x, y \triangleleft g]+[x \triangleleft g, y]+x \triangleleft(y \triangleright g)-y \triangleleft(x \triangleright g) .}
\end{aligned}
$$

Let $(\mathfrak{g}, \mathfrak{h}, \triangleleft, \triangleright)$ be a matched pair of Lie algebras. Then $\mathfrak{g} \bowtie \mathfrak{h}:=\mathfrak{g} \times \mathfrak{h}$, as a vector space, is a Lie algebra with the bracket defined by

$$
\{(g, x),(h, y)\}:=([g, h]+x \triangleright h-y \triangleright g,[x, y]+x \triangleleft h-y \triangleleft g)
$$

for all $g, h \in \mathfrak{g}$ and $x, y \in \mathfrak{h}$, called the bicrossed product associated to the matched pair $(\mathfrak{g}, \mathfrak{h}, \triangleleft, \triangleright)$. Any bicrossed product $\mathfrak{g} \bowtie \mathfrak{h}$ factorizes through $\mathfrak{g}=\mathfrak{g} \times\{0\}$ and $\mathfrak{h}=\{0\} \times \mathfrak{h}$; the converse also holds [17, Proposition 8.3.2]: if a Lie algebra $\mathfrak{L}$ factorizes through $\mathfrak{g}$ and $\mathfrak{h}$, then there exist an isomorphism of Lie algebras $\mathfrak{L} \cong \mathfrak{g} \bowtie \mathfrak{h}$, where $\mathfrak{g} \bowtie \mathfrak{h}$ is the bicrossed product associated to the matched pair $(\mathfrak{g}, \mathfrak{h}, \triangleleft, \triangleright)$ whose actions are constructed from the unique decomposition

$$
[x, g]=x \triangleright g+x \triangleleft g \in \mathfrak{g}+\mathfrak{h}
$$

for all $x \in \mathfrak{h}$ and $g \in \mathfrak{g}$. The matched pair $(\mathfrak{g}, \mathfrak{h}, \triangleleft, \triangleright)$ defined by (2.1) is called the canonical matched pair associated to the factorization $\mathfrak{L}=\mathfrak{g}+\mathfrak{h}$.

Remark 2.1. Over the complex numbers $\mathbb{C}$, an equivalent description for the factorization of a Lie algebra $\mathfrak{L}$ through two Lie subalgebras is given in [5, Definition 2.1] and in [6, Proposition 2.2], in terms of complex product structures of $\mathfrak{L}$, i.e. linear maps $f: \mathfrak{L} \rightarrow \mathfrak{L}$ such that $f \neq \pm \mathrm{Id}, f^{2}=f$ satisfying the integrability conditions

$$
f([x, y])=[f(x), y]+[x, f(y)]-f([f(x), f(y)])
$$

for all $x, y \in \mathfrak{L}$. The linear map $f: \mathfrak{g} \bowtie \mathfrak{h} \rightarrow \mathfrak{g} \bowtie \mathfrak{h}, f(g, h):=(g,-h)$ is a complex product structure on any bicrossed product $\mathfrak{g} \bowtie \mathfrak{h}$. Conversely, if $f$ is a complex product structure on $\mathfrak{L}$, then $\mathfrak{L}$ factorizes through two Lie subalgebras $\mathfrak{L}=\mathfrak{L}_{+}+\mathfrak{L}_{-}$, where $\mathfrak{L}_{ \pm}$denotes the eigenspace corresponding to the eigenvalue \pm 1 of $f$, that is $\mathfrak{L} \cong \mathfrak{L}_{+} \bowtie \mathfrak{L}_{-}$.

Let $(\mathfrak{g}, \mathfrak{h}, \triangleleft, \triangleright)$ be a matched pair of Lie algebras. A linear map $r: \mathfrak{h} \rightarrow \mathfrak{g}$ is called a deformation map [3, Definition 4.1] of the matched pair $(\mathfrak{g}, \mathfrak{h}, \triangleright, \triangleleft)$ if the following compatibility holds for any $x, y \in \mathfrak{h}$

$$
r([x, y])-[r(x), r(y)]=r(y \triangleleft r(x)-x \triangleleft r(y))+x \triangleright r(y)-y \triangleright r(x) .
$$


We denote by $\mathcal{D} \mathcal{M}(\mathfrak{h}, \mathfrak{g} \mid(\triangleright, \triangleleft))$ the set of all deformation maps of the matched pair $(\mathfrak{g}, \mathfrak{h}, \triangleright, \triangleleft)$. If $r \in \mathcal{D} \mathcal{M}(\mathfrak{h}, \mathfrak{g} \mid(\triangleright, \triangleleft))$ then $\mathfrak{h}_{r}:=\mathfrak{h}$, as a vector space, with the new bracket defined for any $x, y \in \mathfrak{h}$ by

$$
[x, y]_{r}:=[x, y]+x \triangleleft r(y)-y \triangleleft r(x)
$$

is a Lie algebra called the $r$-deformation of $\mathfrak{h}$. A Lie algebra $\overline{\mathfrak{h}}$ is a complement of $\mathfrak{g} \cong \mathfrak{g} \times\{0\}$ in the bicrossed product $\mathfrak{g} \bowtie \mathfrak{h}$ if and only if $\overline{\mathfrak{h}} \cong \mathfrak{h}_{r}$, for some deformation map $r \in \mathcal{D} \mathcal{M}(\mathfrak{h}, \mathfrak{g} \mid(\triangleright, \triangleleft))$ [3, Theorem 4.3].

\section{$3 \quad$ The case of perfect Lie algebras}

Computing all matched pairs between two given Lie algebras $\mathfrak{g}$ and $\mathfrak{h}$ and classifying all associated bicrossed products $\mathfrak{g} \bowtie \mathfrak{h}$ is a challenging problem. In the case when $\mathfrak{g}:=k=k_{0}$, the Abelian Lie algebra of dimension 1, they are parameterized by the set TwDer(h) of all twisted derivations of the Lie algebra $\mathfrak{h}$ as defined in [4, Definition 4.2]: a twisted derivation of $\mathfrak{h}$ is a pair $(\lambda, \Delta)$ consisting of two linear maps $\lambda: \mathfrak{h} \rightarrow k$ and $\Delta: \mathfrak{h} \rightarrow \mathfrak{h}$ such that for any $g, h \in \mathfrak{h}$

$$
\lambda([g, h])=0, \quad \Delta([g, h])=[\Delta(g), h]+[g, \Delta(h)]+\lambda(g) \Delta(h)-\lambda(h) \Delta(g) .
$$

TwDer $(\mathfrak{h})$ contains the usual space of derivations $\operatorname{Der}(\mathfrak{h})$ via the canonical embedding $\operatorname{Der}(\mathfrak{h}) \hookrightarrow$ $\operatorname{TwDer}(\mathfrak{h}), D \mapsto(0, D)$, which is an isomorphism if $\mathfrak{h}$ is a perfect Lie algebra (i.e. $\mathfrak{h}=[\mathfrak{h}, \mathfrak{h}])$. As a special case of [4, Proposition 4.4 and Remark 4.5] we have:

Proposition 3.1. Let $\mathfrak{h}$ be a Lie algebra. Then there exists a bijection between the set of all matched pairs $\left(k_{0}, \mathfrak{h}, \triangleleft, \triangleright\right)$ and the space $\operatorname{TwDer}(\mathfrak{h})$ of all twisted derivations of $\mathfrak{h}$ given such that the matched pair $\left(k_{0}, \mathfrak{h}, \triangleleft, \triangleright\right)$ corresponding to $(\lambda, \Delta) \in \operatorname{TwDer}(\mathfrak{h})$ is defined by

$$
h \triangleright a=a \lambda(h), \quad h \triangleleft a=a \Delta(h)
$$

for all $h \in \mathfrak{h}$ and $a \in k=k_{0}$. The bicrossed product $k_{0} \bowtie \mathfrak{h}$ associated to the matched pair (3.2) is denoted by $\mathfrak{h}_{(\lambda, \Delta)}$ and has the bracket given for any $a, b \in k$ and $x, y \in \mathfrak{h}$ by

$$
\{(a, x),(b, y)\}:=(b \lambda(x)-a \lambda(y),[x, y]+b \Delta(x)-a \Delta(y)) .
$$

A Lie algebra $\mathfrak{L}$ contains $\mathfrak{h}$ as a subalgebra of codimension 1 if and only if $\mathfrak{L}$ is isomorphic to $\mathfrak{h}_{(\lambda, \Delta)}$, for some $(\lambda, \Delta) \in \operatorname{TwDer}(\mathfrak{h})$.

Suppose $\left\{e_{i} \mid i \in I\right\}$ is a basis for the Lie algebra $\mathfrak{h}$. Then, $\mathfrak{h}_{(\lambda, \Delta)}$ has $\left\{F, e_{i} \mid i \in I\right\}$ as a basis and the bracket given for any $i \in I$ by

$$
\left[e_{i}, F\right]=\lambda\left(e_{i}\right) F+\Delta\left(e_{i}\right), \quad\left[e_{i}, e_{j}\right]=\left[e_{i}, e_{j}\right]_{\mathfrak{h}},
$$

where $[-,-]_{\mathfrak{h}}$ is the bracket on $\mathfrak{h}$. Above we identify $e_{i}=\left(0, e_{i}\right)$ and denote $F=(1,0)$ in the bicrossed product $k_{0} \bowtie \mathfrak{h}$. Classifying the Lie algebras $\mathfrak{h}_{(\lambda, \Delta)}$ is a difficult task. In what follows we deal with this problem for a perfect Lie algebra $\mathfrak{h}$ : in this case TwDer $(\mathfrak{h})=\{0\} \times \operatorname{Der}(\mathfrak{h})$ and we denote by $\mathfrak{h}_{(\Delta)}=\mathfrak{h}_{(0, \Delta)}$, for any $\Delta \in \operatorname{Der}(\mathfrak{h})$.

Theorem 3.2. Let $\mathfrak{h}$ be a perfect Lie algebra and $\Delta, \Delta^{\prime} \in \operatorname{Der}(\mathfrak{h})$. Then there exists a bijection between the set of all morphisms of Lie algebras $\varphi: \mathfrak{h}_{(\Delta)} \rightarrow \mathfrak{h}_{\left(\Delta^{\prime}\right)}$ and the set of all triples $(\alpha, h, v) \in k \times \mathfrak{h} \times \operatorname{Hom}_{\text {Lie }}(\mathfrak{h}, \mathfrak{h})$ satisfying the following compatibility condition for all $x \in \mathfrak{h}$

$$
v(\Delta(x))-\alpha \Delta^{\prime}(v(x))=[v(x), h] .
$$


The bijection is given such that the Lie algebra map $\varphi=\varphi_{(\alpha, h, v)}$ corresponding to $(\alpha, h, v)$ is given by the formula

$$
\varphi: \mathfrak{h}_{(\Delta)} \rightarrow \mathfrak{h}_{\left(\Delta^{\prime}\right)}, \quad \varphi(a, x)=(a \alpha, a h+v(x))
$$

for all $(a, x) \in \mathfrak{h}_{(\Delta)}=k_{0} \bowtie \mathfrak{h}$. Furthermore, $\varphi=\varphi_{(\alpha, h, v)}$ is an isomorphism of Lie algebras if and only if $\alpha \neq 0$ and $v \in \operatorname{Aut}_{\text {Lie }}(\mathfrak{h})$.

Proof. Any linear map $\varphi: k \times \mathfrak{h} \rightarrow k \times \mathfrak{h}$ is uniquely determined by a quadruple $(\alpha, h, \beta, v)$, where $\alpha \in k, h \in \mathfrak{h}$ and $\beta: \mathfrak{h} \rightarrow k, v: \mathfrak{h} \rightarrow \mathfrak{h}$ are $k$-linear maps such that

$$
\varphi(a, x)=\varphi_{(\alpha, h, \beta, v)}=(a \alpha+\beta(x), a h+v(x)) .
$$

We will prove that $\varphi$ defined above is a Lie algebra map if and only if $\beta$ is the trivial map, $v$ is a Lie algebra map and (3.4) holds. It is enough to test the compatibility

$$
\varphi([(a, x),(b, y)])=[\varphi(a, x), \varphi(b, y)]
$$

for all generators of $\mathfrak{h}_{(\Delta)}=k \times \mathfrak{h}$, i.e. elements of the form $(1,0)$ and $(0, x)$, for all $x \in \mathfrak{h}$. Moreover, since $\mathfrak{h}$ is perfect (i.e. $\lambda=0$ ) the bracket on $\mathfrak{h}_{(\Delta)}$ given by (3.3) takes the form: $\{(a, x),(b, y)\}=(0,[x, y]+b \Delta(x)-a \Delta(y))$. Using this formula we obtain that (3.5) holds for $(0, x)$ and $(0, y)$ if and only if

$$
\beta([x, y])=0, \quad v([x, y])=[v(x), v(y)]+\beta(y) \Delta(v(x))-\beta(x) \Delta(v(y)) .
$$

As $\mathfrak{h}$ is perfect these two conditions are equivalent to the fact that $\beta=0$ and $v$ is a Lie algebra map. Finally, as $\beta=0$, we can easily show that $(3.5)$ holds in $(1,0)$ and $(0, x)$ if and only if (3.4) holds. Thus, we have obtained that $\varphi$ is a Lie algebra map if and only if $v$ is a Lie algebra map, $\beta=0$ and (3.4) holds. In what follows we denote by $\varphi_{(\alpha, h, v)}$ the Lie algebra map corresponding to a quadruple $(\alpha, h, \beta, v)$ with $\beta=0$. Suppose first that $\varphi:=\varphi_{(\alpha, h, v)}$ is a Lie algebra isomorphism. Then, there exists a Lie algebra map $\bar{\varphi}:=\varphi_{(\gamma, g, w)}: \mathfrak{h}_{\left(\Delta^{\prime}\right)} \rightarrow \mathfrak{h}_{(\Delta)}$ such that $\varphi \circ \bar{\varphi}(a, x)=\bar{\varphi} \circ \varphi(a, x)=(a, x)$ for all $a \in k, x \in \mathfrak{h}$. Thus, for all $a \in k$ and $x \in \mathfrak{h}$, we have

$$
a \alpha \gamma=a, \quad a \gamma+v(a g)+v(w(x))=x=a \alpha g+w(a h)+w(v(x)) .
$$

By the first part of (3.6) for $a=1$ we obtain $\alpha \gamma=1$ and thus $\alpha \neq 0$ while the second part of (3.6) for $a=0$ implies $v$ bijective. To end with, assume that $\alpha \neq 0$ and $v \in$ Aut $_{\text {Lie }}(\mathfrak{h})$. Then, it is straightforward to see that $\varphi=\varphi_{(\alpha, h, v)}$ is an isomorphism with the inverse given by $\varphi^{-1}:=\varphi_{\left(\alpha^{-1},-\alpha^{-1} v^{-1}(h), v^{-1}\right)}$.

Let $k^{*}$ be the units group of $k$ and $(\mathfrak{h},+)$ the underlying Abelian group of the Lie algebra $\mathfrak{h}$. Then the map given for any $\alpha \in k^{*}, v \in \operatorname{Aut}_{\text {Lie }}(\mathfrak{h})$ and $h \in \mathfrak{h}$ by

$$
\varphi: k^{*} \times \operatorname{Aut}_{\text {Lie }}(\mathfrak{h}) \rightarrow \operatorname{Aut}_{\mathrm{Gr}}(\mathfrak{h},+), \quad \varphi(\alpha, v)(h):=\alpha^{-1} v(h)
$$

is a morphism of groups. Thus, we can construct the semidirect product of groups $\mathfrak{h} \ltimes_{\varphi}\left(k^{*} \times\right.$ $\left.\operatorname{Aut}_{\text {Lie }}(\mathfrak{h})\right)$ associated to $\varphi$. The next result shows that $\operatorname{Aut}_{\text {Lie }}\left(\mathfrak{h}_{(\Delta)}\right)$ is isomorphic to a certain subgroup of the semidirect product of groups $\mathfrak{h} \ltimes_{\varphi}\left(k^{*} \times \operatorname{Aut}_{\text {Lie }}(\mathfrak{h})\right)$.

Corollary 3.3. Let $\mathfrak{h}$ be a perfect Lie algebra and $\Delta, \Delta^{\prime} \in \operatorname{Der}(\mathfrak{h})$. Then the Lie algebras $\mathfrak{h}_{(\Delta)}$ and $\mathfrak{h}_{\left(\Delta^{\prime}\right)}$ are isomorphic if and only if there exists a triple $(\alpha, h, v) \in k^{*} \times \mathfrak{h} \times \operatorname{Aut}_{\text {Lie }}(\mathfrak{h})$ such that $v \circ \Delta-\alpha \Delta^{\prime} \circ v=[v(-), h]$. Furthermore, there exists an isomorphism of groups

$$
\operatorname{Aut}_{\text {Lie }}\left(\mathfrak{h}_{(\Delta)}\right) \cong \mathcal{G}(\mathfrak{h}, \Delta):=\left\{(\alpha, h, v) \in k^{*} \times \mathfrak{h} \times \operatorname{Aut}_{\text {Lie }}(\mathfrak{h}) \mid v \circ \Delta-\alpha \Delta \circ v=[v(-), h]\right\},
$$


where $\mathcal{G}(\mathfrak{h}, \Delta)$ is a group with respect to the following multiplication

$$
(\alpha, h, v) \cdot(\beta, g, w):=(\alpha \beta, \beta h+v(g), v \circ w)
$$

for all $(\alpha, h, v),(\beta, g, w) \in \mathcal{G}(\mathfrak{h}, \Delta)$. Moreover, the canonical map

$$
\mathcal{G}(\mathfrak{h}, \Delta) \longrightarrow \mathfrak{h} \ltimes_{\varphi}\left(k^{*} \times \operatorname{Aut}_{\text {Lie }}(\mathfrak{h})\right), \quad(\alpha, h, v) \mapsto\left(\alpha^{-1} h,(\alpha, v)\right)
$$

is an injective morphism of groups.

Proof. The first part follows trivially from Theorem 3.2. Consider now $\gamma, \psi \in \operatorname{Aut}_{\text {Lie }}\left(\mathfrak{h}_{(\Delta)}\right)$. Using again Theorem 3.2, we can find $(\alpha, h, v),(\beta, g, w) \in k^{*} \times \mathfrak{h} \times \operatorname{Aut}_{\text {Lie }}(\mathfrak{h})$ such that $\gamma=\varphi_{(\alpha, h, v)}$ and $\psi=\varphi_{(\beta, g, w)}$. Then, for all $a \in k, x \in \mathfrak{h}$ we have

$$
\begin{aligned}
\varphi_{(\alpha, h, v)} \circ \varphi_{(\beta, g, w)}(a, x) & =\varphi_{(\alpha, h, v)}(a \beta, a g+w(x))=(\alpha \beta a, a \beta h+a v(g)+v \circ w(x)) \\
& =\varphi_{(\alpha \beta, \beta h+v(g), v \circ w)}(a, x) .
\end{aligned}
$$

Thus, $\operatorname{Aut}_{\text {Lie }}\left(\mathfrak{h}_{(\Delta)}\right)$ is isomorphic to $\mathcal{G}(\mathfrak{h}, \Delta)$ with the multiplication given by (3.7). The last assertion follows by a routine computation.

Remark 3.4. Let $\Delta=\left[x_{0},-\right]$ be an inner derivation of a perfect Lie algebra $\mathfrak{h}$. Then the group $\operatorname{Aut}_{\text {Lie }}\left(\mathfrak{h}_{\left(\left[x_{0},-\right]\right)}\right)$ admits a simpler description as follows

$$
\mathcal{G}\left(\mathfrak{h},\left[x_{0},-\right]\right)=\left\{(\alpha, h, v) \in k^{*} \times \mathfrak{h} \times \operatorname{Aut}_{\text {Lie }}(\mathfrak{h}) \mid v\left(x_{0}\right)-\alpha x_{0}+h \in \mathrm{Z}(\mathfrak{h})\right\},
$$

where $\mathbf{Z}(\mathfrak{h})$ is the center of $\mathfrak{h}$. Assume in addition that $\mathfrak{h}$ has trivial center, i.e. $Z(\mathfrak{h})=\{0\}$; it follows that there exists an isomorphism of groups

$$
\operatorname{Aut}_{\text {Lie }}\left(\mathfrak{h}_{\left(\left[x_{0},-\right]\right)}\right) \cong k^{*} \times \operatorname{Aut}_{\text {Lie }}(\mathfrak{h}),
$$

since in this case any element $h$ from a triple $(\alpha, h, v) \in \mathcal{G}\left(\mathfrak{h},\left[x_{0},-\right]\right)$ must be equal to $\alpha x_{0}-v\left(x_{0}\right)$. Moreover, in this context, the multiplication given by (3.7) is precisely that of a direct product of groups.

A Lie algebra $\mathfrak{h}$ is called complete (see [14, 22] for examples and structural results on this class of Lie algebras) if $\mathfrak{h}$ has trivial center and any derivation is inner. A complete and perfect Lie algebra is called sympathetic [7]: semisimple Lie algebras over a field of characteristic zero are sympathetic and there exists a sympathetic non-semisimple Lie algebra in dimension 25. For sympathetic Lie algebras, Theorem 3.2 takes the following form which considerably improves [4, Corollary 4.10], where the classification is made only up to an isomorphism of Lie algebras which acts as identity on $\mathfrak{h}$.

Corollary 3.5. Let $\mathfrak{h}$ be a sympathetic Lie algebra. Then up to an isomorphism of Lie algebras there exists only one Lie algebra that contains $\mathfrak{h}$ as a Lie subalgebra of codimension one, namely the direct product $k_{0} \times \mathfrak{h}$ of Lie algebras. Furthermore, there exists an isomorphism of groups $\operatorname{Aut}_{\text {Lie }}\left(k_{0} \times \mathfrak{h}\right) \cong k^{*} \times \operatorname{Aut}_{\text {Lie }}(\mathfrak{h})$.

Proof. Since $\mathfrak{h}$ is perfect any Lie algebra that contains $\mathfrak{h}$ as a Lie subalgebra of codimension 1 is isomorphic to $\mathfrak{h}_{(D)}$, for some $D \in \operatorname{Der}(\mathfrak{h})$. As $\mathfrak{h}$ is also complete, any derivation is inner. For an arbitrary derivation $D=[d,-]$ we can prove that $\mathfrak{h}_{(D)} \cong \mathfrak{h}_{(0)}$, where $0=[0,-]$ is the trivial derivation and moreover $\mathfrak{h}_{(0)}$ is just the direct product of Lie algebras $k_{0} \times \mathfrak{h}$. Indeed, by taking $(\alpha, h, v):=\left(1,-d, \operatorname{Id}_{\mathfrak{h}}\right)$ one can see that relation (3.4) holds for $D=[d,-]$ and $D^{\prime}=[0,-]$, that is $\mathfrak{h}_{(D)} \cong \mathfrak{h}_{(0)}$. The final part follows from Remark 3.4. 
Remark 3.6. Let $\mathfrak{h}$ be a perfect Lie algebra with a basis $\left\{e_{i} \mid i \in I\right\}, \Delta \in \operatorname{Der}(\mathfrak{h})$ a given derivation and consider the extension $k_{0} \subseteq \mathfrak{h}_{(\Delta)}=k_{0} \bowtie \mathfrak{h}_{(\Delta)}$. In order to determine all complements of $k_{0}$ in $\mathfrak{h}_{(\Delta)}$ we have to describe the set of all deformation maps $r: \mathfrak{h} \rightarrow k_{0}$ of the matched pair (3.2). A deformation map is completely determined by a family of scalars $(a)_{i \in I}$ satisfying the following compatibility condition for any $i, j \in I$

$$
r\left(\left[e_{i}, e_{j}\right]_{\mathfrak{h}}\right)=r\left(a_{i} \Delta\left(e_{j}\right)-a_{j} \Delta\left(e_{i}\right)\right)
$$

via the relation $r\left(e_{i}\right)=a_{i}$. For such an $r=\left(a_{i}\right)_{i \in I}$, the $r$-deformation of $\mathfrak{h}$ is the Lie algebra $\mathfrak{h}_{r}$ having $\left\{e_{i} \mid i \in I\right\}$ as a basis and the bracket defined for any $i, j \in I$ by

$$
\left[e_{i}, e_{j}\right]_{r}=\left[e_{i}, e_{j}\right]_{\mathfrak{h}}+a_{j} \Delta\left(e_{i}\right)-a_{i} \Delta\left(e_{j}\right)
$$

Any complement of $k_{0}$ in $\mathfrak{h}_{(\Delta)}$ is isomorphic to such an $\mathfrak{h}_{r}$. An explicit example in dimension 5 is given below.

Example 3.7. Let $k$ be a field of characteristic $\neq 2$ and $\mathfrak{h}$ the perfect 5 -dimensional Lie algebra with a basis $\left\{e_{1}, e_{2}, e_{3}, e_{4}, e_{5}\right\}$ and bracket given by

$$
\begin{aligned}
& {\left[e_{1}, e_{2}\right]=e_{3}, \quad\left[e_{1}, e_{3}\right]=-2 e_{1}, \quad\left[e_{1}, e_{5}\right]=\left[e_{3}, e_{4}\right]=e_{4},} \\
& {\left[e_{2}, e_{3}\right]=2 e_{2}, \quad\left[e_{2}, e_{4}\right]=e_{5}, \quad\left[e_{3}, e_{5}\right]=-e_{5} \text {. }}
\end{aligned}
$$

By a straightforward computation it can be proved that the space of derivations $\operatorname{Der}(\mathfrak{h})$ coincides with the space of all matrices from $\mathcal{M}_{5}(k)$ of the form

$$
A=\left(\begin{array}{ccccc}
a_{1} & 0 & -2 a_{4} & 0 & 0 \\
0 & -a_{1} & -2 a_{2} & 0 & 0 \\
a_{2} & a_{4} & 0 & 0 & 0 \\
a_{3} & 0 & a_{5} & a_{6} & a_{4} \\
0 & a_{5} & -a_{3} & -a_{2} & \left(a_{6}-a_{1}\right)
\end{array}\right)
$$

for all $a_{1}, \ldots, a_{6} \in k$. Thus $\mathfrak{h}$ is not complete since $\operatorname{Der}(\mathfrak{h})$ has dimension 6 . One can show easily that the derivation $\Delta:=e_{11}-e_{41}-e_{22}+e_{53}-e_{44}-2 e_{55}$ is not inner, where $e_{i j} \in \mathcal{M}_{n}(k)$ is the matrix having 1 in the $(i, j)^{\text {th }}$ position and zeros elsewhere. For the derivation $\Delta$ we consider the extension $k_{0} \subseteq k_{0} \bowtie \mathfrak{h}=\mathfrak{h}_{(\Delta)}$ and we will describe all the complements of $k_{0}$ in $\mathfrak{h}_{(\Delta)}$. By a routine computation it can be seen that $r: \mathfrak{h} \rightarrow k_{0}$ is a deformation map of the matched pair (3.2) if and only if $r:=0$ (the trivial map) or $r$ is given by

$$
r\left(e_{1}\right):=a, \quad r\left(e_{2}\right):=-a^{-1}, \quad r\left(e_{3}\right)=2, \quad r\left(e_{4}\right)=r\left(e_{5}\right)=0
$$

for some $a \in k^{*}$. Thus a Lie algebra $\mathfrak{C}$ is a complement of $k_{0}$ in $\mathfrak{h}_{(\Delta)}$ if and only if $\mathfrak{C} \cong \mathfrak{h}$ or $\mathfrak{C} \cong \mathfrak{h}_{a}$, where $\mathfrak{h}_{a}$ is the 5 -dimensional Lie algebra with basis $\left\{e_{1}, e_{2}, e_{3}, e_{4}, e_{5}\right\}$ and bracket given by

$$
\begin{aligned}
& {\left[e_{1}, e_{2}\right]_{a}:=-a^{-1} e_{1}+a e_{2}+e_{3}+a^{-1} e_{4}, \quad\left[e_{1}, e_{3}\right]_{a}:=-2 e_{4}-a e_{5}, \quad\left[e_{1}, e_{4}\right]_{a}:=a e_{4},} \\
& {\left[e_{1}, e_{5}\right]_{a}:=e_{4}+2 a e_{5}, \quad\left[e_{2}, e_{3}\right]_{a}:=a^{-1} e_{5}, \quad\left[e_{2}, e_{4}\right]_{a}:=e_{5}-a^{-1} e_{4},} \\
& {\left[e_{2}, e_{5}\right]_{a}:=-2 a^{-1} e_{5}, \quad\left[e_{3}, e_{4}\right]_{a}:=3 e_{4}, \quad\left[e_{3}, e_{5}\right]_{a}:=3 e_{5}}
\end{aligned}
$$

for any $a \in k^{*}$. Remark that none of the matched pair deformations $\mathfrak{h}_{a}$ of the Lie algebra $\mathfrak{h}$ is perfect since the dimension of the derived algebra $\left[\mathfrak{h}_{a}, \mathfrak{h}_{a}\right]$ is equal to 3 . 


\section{The non-perfect case}

In Section 3 we have described and classified all bicrossed products $k_{0} \bowtie \mathfrak{h}$ for a perfect Lie algebra $\mathfrak{h}$; furthermore, Remark 3.6 and Example 3.7 describe all complements of $k_{0}$ in a given bicrossed product $k_{0} \bowtie \mathfrak{h}$. In this section we approach the same questions for a given non-perfect Lie algebra $\mathfrak{h}:=\mathfrak{l}(2 n+1, k)$, where $\mathfrak{l}(2 n+1, k)$ is the $(2 n+1)$-dimensional Lie algebra with basis $\left\{E_{i}, F_{i}, G \mid i=1, \ldots, n\right\}$ and bracket given for any $i=1, \ldots, n$ by

$$
\left[E_{i}, G\right]:=E_{i}, \quad\left[G, F_{i}\right]:=F_{i} .
$$

First, we shall describe all bicrossed products $k_{0} \bowtie \mathfrak{l}(2 n+1, k)$ : they will explicitly describe all Lie algebras which contain $\mathfrak{l}(2 n+1, k)$ as a subalgebra of codimension 1 . Then, as the second step, we shall find all $r$-deformations of the Lie algebra $\mathfrak{l}(2 n+1, k)$, for two given extensions $k_{0} \subseteq k_{0} \bowtie \mathfrak{l}(2 n+1, k)$. Based on Proposition 3.1 we have to compute first the space $\operatorname{TwDer}(\mathfrak{l}(2 n+1, k))$ of all twisted derivations.

Proposition 4.1. There exists a bijection between $\operatorname{TwDer}(\mathfrak{l}(2 n+1, k))$ and the set of all matrices $\left(A, B, C, D, \lambda_{0}, \delta\right) \in \mathrm{M}_{n}(k)^{4} \times k \times k^{2 n+1}$ satisfying the following conditions

$$
\lambda_{0} A=-\delta_{2 n+1} I_{n}, \quad\left(2+\lambda_{0}\right) B=0, \quad\left(2-\lambda_{0}\right) C=0, \quad \lambda_{0} D=\delta_{2 n+1} I_{n},
$$

where $\delta=\left(\delta_{1}, \ldots, \delta_{2 n+1}\right) \in k^{2 n+1}$. The bijection is given such that the twisted derivation $(\lambda, \Delta) \in \operatorname{TwDer}(\mathfrak{l}(2 n+1, k))$ associated to $\left(A, B, C, D, \lambda_{0}, \delta\right)$ is given by

$$
\begin{aligned}
& \lambda\left(E_{i}\right)=\lambda\left(F_{i}\right):=0, \quad \lambda(G):=\lambda_{0}, \\
& \Delta:=\left(\begin{array}{ccc}
A & B & \delta_{1} \\
C & D: & \\
0 & 0 & \delta_{2 n+1}
\end{array}\right) .
\end{aligned}
$$

$\mathcal{T}(n)$ denotes the set of all $\left(A, B, C, D, \lambda_{0}, \delta\right) \in \mathrm{M}_{n}(k)^{4} \times k \times k^{2 n+1}$ satisfying (4.1).

Proof. The first compatibility condition (3.1) shows that a linear map $\lambda: \mathfrak{l}(2 n+1, k) \rightarrow k$ of a twisted derivation $(\lambda, D)$ must have the form given by (4.2), for some $\lambda_{0} \in k$. We shall fix such a map for a given $\lambda_{0} \in k$. We write down the linear map $\Delta: \mathfrak{l}(2 n+1, k) \rightarrow \mathfrak{l}(2 n+1, k)$ as a matrix associated to the basis $\left\{E_{1}, \ldots, E_{n}, F_{1}, \ldots, F_{n}, G\right\}$ of $\mathfrak{l}(2 n+1, k)$, as follows

$$
\Delta=\left(\begin{array}{ccc}
A & B & d_{1,2 n+1} \\
C & D: & \\
d_{2 n+1,1} . . d_{2 n+1,2 n+1} & &
\end{array}\right)
$$

for some matrices $A, B, C, D \in \mathrm{M}_{n}(k)$ and some scalars $d_{i, j} \in k$, for all $i, j=1, \ldots, 2 n+1$. We denote $A=\left(a_{i j}\right), B=\left(b_{i j}\right), C=\left(c_{i j}\right), D=\left(d_{i j}\right)$. It remains to check the compatibility condition (3.1) for $\Delta$, i.e.

$$
\Delta([g, h])=[\Delta(g), h]+[g, \Delta(h)]+\lambda(g) \Delta(h)-\lambda(h) \Delta(g)
$$

for all $g \neq h \in\left\{E_{1}, \ldots, E_{n}, F_{1}, \ldots, F_{n}, G\right\}$. As this is a routinely straightforward computation we will only indicate the main steps of the proof. We can easily see that the compatibility condition (3.1) holds for $(g, h)=\left(E_{i}, E_{j}\right)$ if and only if $d_{2 n+1, i}=0$, for all $i=1, \ldots, n$. In the same way $(3.1)$ holds for $(g, h)=\left(F_{i}, F_{j}\right)$ if and only if $d_{2 n+1, n+i}=0$, for all $i=1, \ldots, n$. This shows that $\Delta$ has the form (4.3), that is the first $2 n$ entries from the last row of the matrix $\Delta$ are all zeros and we will denote the last column of $D$ by $\left(d_{1,2 n+1}, \ldots, d_{2 n+1,2 n+1}\right)=$ $\delta=\left(\delta_{1}, \ldots, \delta_{2 n+1}\right)$. It follows from here that $(3.1)$ holds trivially for the pair $(g, h)=\left(E_{i}, F_{j}\right)$. 
An easy computation shows that (3.1) holds for $(g, h)=\left(E_{i}, G\right)$ if and only if the following equation holds

$$
\left(1-\lambda_{0}\right)\left(\sum_{j=1}^{n} a_{j, i} E_{j}+\sum_{j=1}^{n} c_{j, i} F_{j}\right)=\sum_{j=1}^{n} a_{j, i} E_{j}-\sum_{j=1}^{n} c_{j, i} F_{j}+\delta_{2 n+1} E_{i},
$$

which is equivalent to $-\lambda_{0} A=\delta_{2 n+1} I_{n}$ and $\left(2-\lambda_{0}\right) C=0$, i.e. the first and the third equations from (4.1). A similar computation shows that (3.1) holds for $(g, h)=\left(G, F_{i}\right)$ if and only if $\left(2+\lambda_{0}\right) B=0$ and $\lambda_{0} D=\delta_{2 n+1} I_{n}$ and the proof is finished.

Let $\mathfrak{l}(2 n+1, k)_{\left(A, B, C, D, \lambda_{0}, \delta\right)}$ be the bicrossed product $k_{0} \bowtie \mathfrak{l}(2 n+1, k)$ associated to the matched pair given by the twisted derivation $\left(A=\left(a_{j i}\right), B=\left(b_{j i}\right), C=\left(c_{j i}\right), D=\left(d_{j i}\right), \lambda_{0}\right.$, $\left.\delta=\left(\delta_{j}\right)\right) \in \mathcal{T}(n)$. From now on we will use the following convention: if one of the elements of the 6 -tuple $\left(A, B, C, D, \lambda_{0}, \delta\right)$ is equal to 0 then we will omit it when writing down the Lie algebra $\mathfrak{l}(2 n+1, k)_{\left(A, B, C, D, \lambda_{0}, \delta\right)}$. A basis of $\mathfrak{l}(2 n+1, k)_{\left(A, B, C, D, \lambda_{0}, \delta\right)}$ will be denoted by $\left\{E_{i}, F_{i}, G, H \mid i=1, \ldots, n\right\}$ : these Lie algebras can be explicitly described by first computing the set $\mathcal{T}(n)$ and then using Proposition 3.1. Considering the equations (4.1) which define $\mathcal{T}(n)$ a discussion involving the field $k$ and the scalar $\lambda_{0}$ is mandatory. For two sets $X$ and $Y$ we shall denote by $X \sqcup Y$ the disjoint union of $X$ and $Y$. As a conclusion of the above results we obtain:

Theorem 4.2. (1) If $k$ is a field such that $\operatorname{char}(k) \neq 2$ then

$$
\mathcal{T}(n) \cong\left((k \backslash\{0, \pm 2\}) \times k^{2 n+1}\right) \sqcup\left(\mathrm{M}_{n}(k)^{2} \times k^{2 n}\right) \sqcup\left(\mathrm{M}_{n}(k) \times k^{2 n+1}\right) \sqcup\left(\mathrm{M}_{n}(k) \times k^{2 n+1}\right)
$$

and the four families of Lie algebras containing $\mathfrak{l}(2 n+1, k)$ as a subalgebra of codimension 1 are the following:

- the Lie algebra $\mathfrak{l}^{1}(2 n+1, k)_{\left(\lambda_{0}, \delta\right)}$ with the bracket given for any $i=1, \ldots, n$ by

$$
\begin{aligned}
& {\left[E_{i}, G\right]=E_{i}, \quad\left[G, F_{i}\right]=F_{i}, \quad\left[E_{i}, H\right]=-\lambda_{0}^{-1} \delta_{2 n+1} E_{i},} \\
& {\left[F_{i}, H\right]=\lambda_{0}^{-1} \delta_{2 n+1} F_{i}, \quad[G, H]=\lambda_{0} H+\sum_{j=1}^{n} \delta_{j} E_{j}+\sum_{j=1}^{n} \delta_{n+j} F_{j}+\delta_{2 n+1} G}
\end{aligned}
$$

for all $\left(\lambda_{0}, \delta\right) \in(k \backslash\{0, \pm 2\}) \times k^{2 n+1}$.

- the Lie algebra $\mathfrak{l}^{2}(2 n+1, k)_{(A, D, \delta)}$ with the bracket given for any $i=1, \ldots, n$ by

$$
\begin{aligned}
& {\left[E_{i}, G\right]=E_{i}, \quad\left[G, F_{i}\right]=F_{i}, \quad\left[E_{i}, H\right]=\sum_{j=1}^{n} a_{j i} E_{j}} \\
& {\left[F_{i}, H\right]=\sum_{j=1}^{n} d_{j i} F_{j}, \quad[G, H]=\sum_{j=1}^{n} \delta_{j} E_{j}+\sum_{j=1}^{n} \delta_{n+j} F_{j}}
\end{aligned}
$$

for all $\left(A=\left(a_{i j}\right), D=\left(d_{i j}\right), \delta\right) \in \mathrm{M}_{n}(k) \times \mathrm{M}_{n}(k) \times k^{2 n}$.

- the Lie algebra $\mathfrak{l}^{3}(2 n+1, k)_{(C, \delta)}$ with the bracket given for any $i=1, \ldots, n$ by

$$
\begin{aligned}
& {\left[E_{i}, G\right]=E_{i}, \quad\left[G, F_{i}\right]=F_{i}, \quad\left[E_{i}, H\right]=-2^{-1} \delta_{2 n+1} E_{i}+\sum_{j=1}^{n} c_{j i} F_{j},} \\
& {\left[F_{i}, H\right]=2^{-1} \delta_{2 n+1} F_{i}, \quad[G, H]=2 H+\sum_{j=1}^{n} \delta_{j} E_{j}+\sum_{j=1}^{n} \delta_{n+j} F_{j}+\delta_{2 n+1} G}
\end{aligned}
$$

for all $\left(C=\left(c_{i j}\right), \delta\right) \in \mathrm{M}_{n}(k) \times k^{2 n+1}$. 
- the Lie algebra $\mathfrak{l}^{4}(2 n+1, k)_{(B, \delta)}$ with the bracket given for any $i=1, \ldots, n$ by

$$
\begin{aligned}
& {\left[E_{i}, G\right]=E_{i}, \quad\left[G, F_{i}\right]=F_{i}, \quad\left[F_{i}, H\right]=\sum_{j=1}^{n} b_{j i} E_{j}-2^{-1} \delta_{2 n+1} F_{i},} \\
& {\left[E_{i}, H\right]=2^{-1} \delta_{2 n+1} E_{i}, \quad[G, H]=-2 H+\sum_{j=1}^{n} \delta_{j} E_{j}+\sum_{j=1}^{n} \delta_{n+j} F_{j}+\delta_{2 n+1} G}
\end{aligned}
$$

for all $\left(B=\left(b_{i j}\right), \delta\right) \in \mathrm{M}_{n}(k) \times k^{2 n+1}$.

(2) If $\operatorname{char}(k)=2$ then

$$
\mathcal{T}(n) \cong\left(\mathrm{M}_{n}(k)^{4} \times k^{2 n}\right) \sqcup\left(k^{*} \times k^{2 n+1}\right)
$$

and the two families of Lie algebras containing $\mathfrak{l}(2 n+1, k)$ as a subalgebra of codimension 1 are the following:

- the Lie algebra $\mathfrak{l}_{1}(2 n+1, k)_{(A, B, C, D, \delta)}$ with the bracket given for any $i=1, \ldots, n$ by

$$
\begin{array}{ll}
{\left[E_{i}, G\right]=E_{i}, \quad\left[G, F_{i}\right]=F_{i}, \quad\left[E_{i}, H\right]=\sum_{j=1}^{n}\left(a_{j i} E_{j}+c_{j i} F_{j}\right),} \\
{\left[F_{i}, H\right]=\sum_{j=1}^{n}\left(b_{j i} E_{j}+d_{j i} F_{j}\right),} & {[G, H]=\sum_{j=1}^{n} \delta_{j} E_{j}+\sum_{j=1}^{n} \delta_{n+j} F_{j}}
\end{array}
$$

for all $(A, B, C, D, \delta) \in \mathrm{M}_{n}(k)^{4} \times k^{2 n}$.

- the Lie algebra $\mathfrak{l}_{2}(2 n+1, k)_{\left(\lambda_{0}, \delta\right)}$ with the bracket given for any $i=1, \ldots, n$ by

$$
\begin{aligned}
& {\left[E_{i}, G\right]=E_{i}, \quad\left[G, F_{i}\right]=F_{i}, \quad\left[E_{i}, H\right]=-\lambda_{0}^{-1} \delta_{2 n+1} E_{i},} \\
& {\left[F_{i}, H\right]=\lambda_{0}^{-1} \delta_{2 n+1} F_{i}, \quad[G, H]=\lambda_{0} H+\sum_{j=1}^{n} \delta_{j} E_{j}+\sum_{j=1}^{n} \delta_{n+j} F_{j}+\delta_{2 n+1} G}
\end{aligned}
$$

for all $\left(\lambda_{0}, \delta\right) \in k^{*} \times k^{2 n+1}$.

Proof. The proof relies on the use of Propositions 3.1 and 4.1 as well as the equations (4.1) defining $\mathcal{T}(n)$. Besides the discussion on the characteristic of $k$ it is also necessary to consider whether $\lambda_{0}$ belongs to the set $\{0,2,-2\}$. In the case that $\operatorname{char}(k) \neq 2$, the first Lie algebra listed is the bicrossed product which corresponds to the case when $\lambda_{0} \notin\{0,2,-2\}$. In this case, we can easily see that $\left(A, B, C, D, \lambda_{0}, \delta=\left(\delta_{j}\right)\right) \in \mathcal{T}(n)$ if and only if $B=C=0$, $A=-\lambda_{0}^{-1} \delta_{2 n+1} I_{n}$ and $D=\lambda_{0}^{-1} \delta_{2 n+1} I_{n}$. The Lie algebra $\mathfrak{l}^{1}(2 n+1, k)_{\left(\lambda_{0}, \delta\right)}$ is exactly the bicrossed product $k_{0} \bowtie \mathfrak{l}(2 n+1, k)$ corresponding to this twisted derivation. The Lie algebra $\mathfrak{l}^{2}(2 n+1, k)_{(A, D, \delta)}$ is the bicrossed product $k_{0} \bowtie \mathfrak{l}(2 n+1, k)$ corresponding to the case $\lambda_{0}=0$ while the last two Lie algebras are the bicrossed products $k_{0} \bowtie \mathfrak{l}(2 n+1, k)$ associated to the case when $\lambda_{0}=2$ and respectively $\lambda_{0}=-2$.

If the characteristic of $k$ is equal to 2 we distinguish the following two possibilities: the Lie algebra $\mathfrak{l}_{1}(2 n+1, k)_{(A, B, C, D, \delta)}$ is the bicrossed product $k_{0} \bowtie \mathfrak{l}(2 n+1, k)$ associated to $\lambda_{0}=0$ while the Lie algebra $\mathfrak{l}_{2}(2 n+1, k)_{\left(\lambda_{0}, \delta\right)}$ is the same bicrossed product but associated to $\lambda_{0} \neq 0$.

Let $k$ be a field of characteristic $\neq 2$ and $\mathfrak{l}^{1}(2 n+1, k)_{\left(\lambda_{0}, \delta\right)}$ the Lie algebra of Theorem 4.2. In order to keep the computations efficient we will consider $\lambda_{0}:=1$ and $\delta:=(0, \ldots, 0,1)$ and we denote by $L(2 n+2, k):=\mathfrak{l}^{1}(2 n+1, k)_{(1,(0, \ldots, 0,1))}$, the $(2 n+2)$-dimensional Lie algebra having a basis $\left\{E_{i}, F_{i}, G, H \mid i=1, \ldots, n\right\}$ and the bracket defined for any $i=1, \ldots, n$ by

$$
\left[E_{i}, G\right]=E_{i}, \quad\left[G, F_{i}\right]=F_{i}, \quad\left[E_{i}, H\right]=-E_{i}, \quad\left[F_{i}, H\right]=F_{i}, \quad[G, H]=H+G .
$$


We consider the Lie algebra extension $k H \subset L(2 n+2, k)$, where $k H \cong k_{0}$ is the Abelian Lie algebra of dimension 1. Of course, $L(2 n+2, k)$ factorizes through $k H$ and $\mathfrak{l}(2 n+1, k)$, i.e. $L(2 n+2, k)=k H \bowtie \mathfrak{l}(2 n+1, k)$ - the actions $\triangleleft: \mathfrak{l}(2 n+1, k) \times k H \rightarrow \mathfrak{l}(2 n+1, k)$ and $\triangleright: \mathfrak{l}(2 n+1, k) \times k H \rightarrow k H$ of the canonical matched pair are given by

$$
E_{i} \triangleleft H:=-E_{i}, \quad F_{i} \triangleleft H:=F_{i}, \quad G \triangleleft H:=G, \quad G \triangleright H:=H
$$

and all undefined actions are zero. Next we compute the set $\mathcal{D} \mathcal{M}(\mathfrak{l}(2 n+1, k), k H \mid(\triangleright, \triangleleft))$ of all deformation maps of the matched pair $(k H, \mathfrak{l}(2 n+1, k), \triangleright, \triangleleft)$ given by (4.4).

Lemma 4.3. Let $k$ be a field of characteristic $\neq 2$. Then there exists a bijection

$$
\mathcal{D} \mathcal{M}(\mathfrak{l}(2 n+1, k), k H \mid(\triangleright, \triangleleft)) \cong\left(k^{n} \backslash\{0\}\right) \sqcup\left(k^{n} \times k\right) .
$$

The bijection is given such that the deformation map $r=r_{a}: \mathfrak{l}(2 n+1, k) \rightarrow k H$ associated to $a=\left(a_{i}\right) \in k^{n} \backslash\{0\}$ is given by

$$
r\left(E_{i}\right):=a_{i} H, \quad r\left(F_{i}\right):=0, \quad r(G):=H,
$$

while the deformation map $r=r_{(b, c)}: \mathfrak{l}(2 n+1, k) \rightarrow k H$ associated to $\left(b=\left(b_{i}\right), c\right) \in k^{n} \times k$ is given as follows

$$
r\left(E_{i}\right):=0, \quad r\left(F_{i}\right):=b_{i} H, \quad r(G):=c H
$$

for all $i=1, \ldots, n$.

Proof. Any linear map $r: \mathfrak{l}(2 n+1, k) \rightarrow k H$ is uniquely determined by a triple $\left(a=\left(a_{i}\right), b=\right.$ $\left.\left(b_{i}\right), c\right) \in k^{n} \times k^{n} \times k$ via: $r\left(E_{i}\right):=a_{i} H, r\left(F_{i}\right):=b_{i} H$ and $r(G):=c H$, for all $i=1, \ldots, n$. We need to check under what conditions such a map $r=r_{(a, b, c)}$ is a deformation map. Since $k H$ is Abelian, equation (2.2) comes down to

$$
r([x, y])=r(y \triangleleft r(x)-x \triangleleft r(y))+x \triangleright r(y)-y \triangleright r(x),
$$

which needs to be checked for all $x, y \in\left\{E_{i}, F_{i}, G \mid i=1, \ldots, n\right\}$. Notice that (4.7) is symmetrical i.e. if (4.7) is fulfilled for $(x, y)$ then (4.7) is also fulfilled for $(y, x)$. By a routinely computation it can be seen that $r=r_{(a, b, c)}$ is a deformation map if and only if

$$
a_{i} b_{j}=0, \quad(1-c) a_{i}=0
$$

for all $i, j=1, \ldots, n$. Indeed, (4.7) holds for $(x, y)=\left(E_{i}, F_{j}\right)$ if and only if $a_{i} b_{j}=0$ and it holds for $(x, y)=\left(E_{i}, G\right)$ if and only if $a_{i}=a_{i} c$. The other cases left to study are either automatically fulfilled or equivalent to one of the two conditions above. The first condition of (4.8) divides the description of deformation maps into two cases: the first one corresponds to $a=\left(a_{i}\right) \neq 0$ and we automatically have $b=0$ and $c=1$. The second case corresponds to $a:=0$ which implies that (4.8) holds for any $(b, c) \in k^{n} \times k$.

The next result describes all deformations of $\mathfrak{l}(2 n+1, k)$ associated to the canonical matched pair $(k H, \mathfrak{l}(2 n+1, k), \triangleright, \triangleleft)$ given by $(4.4)$.

Proposition 4.4. Let $k$ be a field of characteristic $\neq 2$ and the extension of Lie algebras $k H \subset L(2 n+2, k)$. Then a Lie algebra $\mathfrak{C}$ is a complement of $k H$ in $L(2 n+2, k)$ if and only if $\mathfrak{C}$ is isomorphic to one of the Lie algebras from the three families defined below:

- the Lie algebra $\mathfrak{l}_{(a)}(2 n+1, k)$ having the bracket def ined for any $i=1, \ldots, n$ by

$$
\left[E_{i}, E_{j}\right]_{a}:=a_{i} E_{j}-a_{j} E_{i}, \quad\left[E_{i}, F_{j}\right]_{a}:=-a_{i} F_{j}, \quad\left[E_{i}, G\right]_{a}:=-a_{i} G
$$

for all $a=\left(a_{i}\right) \in k^{n} \backslash\{0\}$. 
- the Lie algebra $\mathfrak{l}_{(b)}^{\prime}(2 n+1, k)$ having the bracket def ined for any $i=1, \ldots, n$ by

$$
\begin{aligned}
& {\left[E_{i}, F_{j}\right]_{b}:=-b_{j} E_{i}, \quad\left[E_{i}, G\right]_{b}:=-E_{i},} \\
& {\left[F_{i}, F_{j}\right]_{b}:=b_{j} F_{i}-b_{i} F_{j}, \quad\left[F_{i}, G\right]_{b}:=F_{i}-b_{i} G}
\end{aligned}
$$

for all $b=\left(b_{i}\right) \in k^{n}$.

- the Lie algebra $\mathfrak{l}_{(b)}^{\prime \prime}(2 n+1, k)$ having the bracket def ined for any $i=1, \ldots, n$ by

$$
\left[E_{i}, F_{j}\right]_{b}:=-b_{j} E_{i}, \quad\left[F_{i}, F_{j}\right]_{b}:=b_{j} F_{i}-b_{i} F_{j}, \quad\left[F_{i}, G\right]_{b}:=-b_{i} G
$$

for all $b=\left(b_{i}\right) \in k^{n}$.

Thus the factorization index $[L(2 n+2, k): k H]^{f}$ is equal to the number of types of isomorphisms of Lie algebras of the set

$$
\left\{\mathfrak{l}_{(a)}(2 n+1, k), \mathfrak{l}_{(b)}^{\prime}(2 n+1, k), \mathfrak{l}_{(b)}^{\prime \prime}(2 n+1, k) \mid a \in k^{n} \backslash\{0\}, b \in k^{n}\right\} .
$$

Proof. $\mathfrak{l}(2 n+1, k)$ is a complement of $k H$ in $L(2 n+2, k)$ and we can write $L(2 n+2, k)=$ $k H \bowtie \mathfrak{l}(2 n+1, k)$, where the bicrossed product is associated to the matched pair given in (4.4). Hence, by [3, Theorem 4.3] any other complement $\mathfrak{C}$ of $k H$ in $L(2 n+2, k)$ is isomorphic to an $r$-deformation of $\mathfrak{l}(2 n+1, k)$, for some deformation map $r: \mathfrak{l}(2 n+1, k) \rightarrow k H$ of the matched pair (4.4). These are described in Lemma 4.3. The Lie algebra $\mathfrak{l}_{(a)}(2 n+1, k)$ is precisely the $r_{a^{-}}$ deformation of $\mathfrak{l}(2 n+1, k)$, where $r_{a}$ is given by (4.5). On the other hand the $r_{(b, c)}$-deformation of $\mathfrak{l}(2 n+1, k)$, where $r_{(b, c)}$ is given by (4.6) for some $\left(b=\left(b_{i}\right), c\right) \in k^{n} \times k$, is the Lie algebra denoted by $\mathfrak{l}_{(b, c)}(2 n+1, k)$ having the bracket given for any $i=1, \ldots, n$ by

$$
\begin{aligned}
& {\left[E_{i}, F_{j}\right]_{(b, c)}:=-b_{j} E_{i}, \quad\left[E_{i}, G\right]_{(b, c)}:=(1-c) E_{i},} \\
& {\left[F_{i}, F_{j}\right]_{(b, c)}:=b_{j} F_{i}-b_{i} F_{j}, \quad\left[F_{i}, G\right]_{(b, c)}:=(c-1) F_{i}-b_{i} G}
\end{aligned}
$$

for all $\left(b=\left(b_{i}\right), c\right) \in k^{n} \times k$. Now, for $c \neq 1$ we can see that $\mathfrak{l}_{(b, c)}(2 n+1, k) \cong \mathfrak{l}_{(b)}^{\prime}(2 n+1, k)$ (by sending $G$ to $\left.(c-1)^{-1} G\right)$ while $\mathfrak{l}_{(b, 1)}(2 n+1, k)=\mathfrak{l}_{(b)}^{\prime \prime}(2 n+1, k)$ and we are done.

Remark 4.5. An attempt to compute $[L(2 n+2, k): k H]^{f}$ for an arbitrary integer $n$ is hopeless. However, one can easily see that $\mathfrak{l}_{(0)}^{\prime}(2 n+1, k)=\mathfrak{l}(2 n+1, k)$ and $\mathfrak{l}_{(0)}^{\prime \prime}(2 n+1, k)=k_{0}^{2 n+1}$, the Abelian Lie algebra of dimension $2 n+1$. Thus, $[L(2 n+2, k): k H]^{f} \geq 2$. The case $n=1$ is presented below.

Example 4.6. Let $k$ be a field of characteristic $\neq 2$ and consider $\{E, F, G\}$ the basis of $\mathfrak{r}(3, k)$ with the bracket given by $[E, G]=E$ and $[G, F]=F$. Then, the factorization index $[L(4, k)$ : $k H]^{f}=3$. More precisely, the isomorphism classes of all complements of $k H$ in $L(4, k)$ are represented by the following three Lie algebras: $\mathfrak{r}(3, k), k_{0}^{3}$ and the Lie algebra $L_{-1}$ having $\{E, F, G\}$ as a basis and the bracket given by

$$
[F, E]=F, \quad[E, G]=-G .
$$

Since $\operatorname{char}(k) \neq 2$ the Lie algebras $\mathfrak{l}(3, k)$ and $L_{-1}$ are not isomorphic [9, Exercise 3.2]. For $a \in k^{*}$ the Lie algebra $\mathfrak{l}_{(a)}(3, k)$ has the bracket given by $[E, F]=-a F$ and $[E, G]=-a G$. Thus, $\mathfrak{l}_{(a)}(3, k) \cong \mathfrak{l}_{(1)}(3, k)$, and the latter is isomorphic to the Lie algebra $L_{-1}$. On the other hand we have: $\mathfrak{l}_{(0)}^{\prime \prime}(3, k)=k_{0}^{3}$ and for $b \neq 0$ we can easily see that $\mathfrak{l}_{(b)}^{\prime \prime}(3, k) \cong \mathfrak{l}_{(1)}^{\prime \prime}(3, k) \cong \mathfrak{l}(3, k)$. Finally, $\mathfrak{l}_{(0)}^{\prime}(3, k)=\mathfrak{l}(3, k)$ and for $b \neq 0$ we have that $\mathfrak{l}_{(b)}^{\prime}(3, k) \cong \mathfrak{l}_{(1)}^{\prime}(3, k)$ - the latter is the Lie algebra having $\left\{f_{1}, f_{2}, f_{3}\right\}$ as a basis and the bracket given by $\left[f_{1}, f_{2}\right]=-f_{1},\left[f_{1}, f_{3}\right]=f_{1}$ and $\left[f_{3}, f_{2}\right]=f_{2}+f_{3}$. This Lie algebra is also isomorphic to $\mathfrak{l}(3, k)$, via the isomorphism which sends $f_{1}$ to $E, f_{3}$ to $G$ and $f_{2}$ to $F-G$. 
Let $k$ be a field of characteristic $\neq 2$ and $\mathfrak{l}^{2}(2 n+1, k)_{(A, D, \delta)}$ the Lie algebra of Theorem 4.2. In order to simplify computations we will assume $A=D:=I_{n}$ and $\delta:=(1,0, \ldots, 0,1)$. Let $\mathfrak{m}(2 n+2, k):=\mathfrak{l}^{2}(2 n+1, k)_{\left(I_{n}, I_{n},(1,0, \ldots, 0,1)\right)}$ be the $(2 n+2)$-dimensional Lie algebra having $\left\{E_{i}, F_{i}, G, H \mid i=1, \ldots, n\right\}$ as a basis and the bracket defined for any $i=1, \ldots, n$ by

$$
\left[E_{i}, G\right]=E_{i}, \quad\left[G, F_{i}\right]=F_{i}, \quad\left[E_{i}, H\right]=E_{i}, \quad\left[F_{i}, H\right]=F_{i}, \quad[G, H]=E_{1}+F_{n} .
$$

We consider the Lie algebra extension $k H \subset \mathfrak{m}(2 n+2, k)$, where $k H \cong k_{0}$ is the Abelian Lie algebra of dimension 1. Of course, $\mathfrak{m}(2 n+2, k)$ factorizes through $k H$ and $\mathfrak{l}(2 n+1, k)$, i.e. $\mathfrak{m}(2 n+2, k)=k H \bowtie \mathfrak{l}(2 n+1, k)$. Moreover, the canonical matched pair $\triangleleft: \mathfrak{l}(2 n+1, k) \times k H \rightarrow$ $\mathfrak{l}(2 n+1, k)$ and $\triangleright: \mathfrak{l}(2 n+1, k) \times k H \rightarrow k H$ associated to this factorization is given as follows:

$$
E_{i} \triangleleft H:=E_{i}, \quad F_{i} \triangleleft H:=F_{i}, \quad G \triangleleft H:=E_{1}+F_{n}
$$

and all undefined actions are zero. In particular, we should notice that the left action $\triangleright$ : $\mathfrak{l}(2 n+1, k) \times k H \rightarrow k H$ is trivial. Next, we describe the set $\mathcal{D} \mathcal{M}(\mathfrak{l}(2 n+1, k), k H \mid(\triangleright, \triangleleft))$ of all deformation maps of the matched pair $(k H, \mathfrak{l}(2 n+1, k), \triangleright, \triangleleft)$ given by (4.10).

Lemma 4.7. Let $k$ be a field of characteristic $\neq 2$. Then there exists a bijection

$$
\mathcal{D} \mathcal{M}(\mathfrak{l}(2 n+1, k), k H \mid(\triangleright, \triangleleft)) \cong\left(k^{n} \backslash\{0\}\right) \sqcup\left(k^{n} \backslash\{0\}\right) \sqcup k .
$$

The bijection is given such that the deformation map $r=r_{a}: \mathfrak{l}(2 n+1, k) \rightarrow k H$ associated to $a=\left(a_{i}\right) \in k^{n} \backslash\{0\}$ is given by

$$
r\left(E_{i}\right):=a_{i} H, \quad r\left(F_{i}\right):=0, \quad r(G):=\left(a_{1}-1\right) H
$$

the deformation map $r=r_{b}: \mathfrak{l}(2 n+1, k) \rightarrow k H$ associated to another $b=\left(b_{i}\right) \in k^{n} \backslash\{0\}$ is given by

$$
r\left(E_{i}\right):=0, \quad r\left(F_{i}\right):=b_{i} H, \quad r(G):=\left(b_{n}+1\right) H,
$$

while the deformation map $r=r_{c}: \mathfrak{l}(2 n+1, k) \rightarrow k H$ associated to $c \in k$ is given by

$$
r\left(E_{i}\right):=0, \quad r\left(F_{i}\right):=0, \quad r(G):=c H
$$

for all $i=1, \ldots, n$.

Proof. Any linear map $r: \mathfrak{l}(2 n+1, k) \rightarrow k H$ is uniquely determined by a triple $\left(a=\left(a_{i}\right)\right.$, $\left.b=\left(b_{i}\right), c\right) \in k^{n} \times k^{n} \times k$ via: $r\left(E_{i}\right):=a_{i} H, r\left(F_{i}\right):=b_{i} H$ and $r(G):=c H$, for all $i=1, \ldots, n$. We only need to check when such a map $r=r_{(a, b, c)}$ is a deformation map. Since $k H$ is the Abelian Lie algebra and the left action $\triangleright: \mathfrak{l}(2 n+1, k) \times k H \rightarrow k H$ is trivial, equation (2.2) comes down to

$$
r([x, y])=r(y \triangleleft r(x)-x \triangleleft r(y)) .
$$

Since (4.14) is symmetrical it is enough to check it only for pairs of the form $\left(E_{i}, E_{j}\right),\left(F_{i}, F_{j}\right)$, $\left(E_{i}, F_{j}\right),\left(E_{i}, G\right)$, and $\left(F_{i}, G\right)$, for all $i, j=1, \ldots, n$. It is straightforward to see that (4.14) is trivially fulfilled for the pairs $\left(E_{i}, E_{j}\right),\left(F_{i}, F_{j}\right)$ and $\left(E_{i}, F_{j}\right)$. Moreover, (4.14) evaluated for $\left(E_{i}, G\right)$ and respectively $\left(F_{i}, G\right)$ yields $a_{i}\left(a_{1}+b_{n}-c-1\right)=0$ and $b_{i}\left(a_{1}+b_{n}-c+1\right)=0$ for all $i=1, \ldots, n$. Therefore, keeping in mind that we work over a field of characteristic $\neq 2$, the triples $\left(a=\left(a_{i}\right), b=\left(b_{i}\right), c\right) \in k^{n} \times k^{n} \times k$ for which $r_{(a, b, c)}$ becomes a deformation map are given as follows: $\left(a=\left(a_{i}\right) \in k^{n} \backslash\{0\}, b=0, c=a_{1}-1\right),\left(a=0, b=\left(b_{i}\right) \in k^{n} \backslash\{0\}, c=b_{n}+1\right)$ and $(a=0, b=0, c \in k)$. The corresponding deformation maps are exactly those listed above. 
The next result describes all deformations of $\mathfrak{l}(2 n+1, k)$ associated to the canonical matched pair $(k H, \mathfrak{l}(2 n+1, k), \triangleright, \triangleleft)$ given by $(4.10)$.

Proposition 4.8. Let $k$ be a field of characteristic $\neq 2$ and the extension of Lie algebras $k H \subset \mathfrak{m}(2 n+2, k)$. Then a Lie algebra $\mathfrak{C}$ is a complement of $k H$ in $\mathfrak{m}(2 n+2, k)$ if and only if $\mathfrak{C}$ is isomorphic to one of the Lie algebras from the three families defined below:

- the Lie algebra $\overline{\mathfrak{l}}_{(a)}(2 n+1, k)$ having the bracket def ined for any $i=1, \ldots, n$ by

$$
\begin{aligned}
& {\left[E_{i}, E_{j}\right]_{a}:=a_{j} E_{i}-a_{i} E_{j}, \quad\left[E_{i}, F_{j}\right]_{a}:=-a_{i} F_{j},} \\
& {\left[E_{i}, G\right]_{a}:=a_{1} E_{i}-a_{i}\left(E_{1}+F_{n}\right), \quad\left[G, F_{i}\right]_{a}:=\left(2-a_{1}\right) F_{i}}
\end{aligned}
$$

for all $a=\left(a_{i}\right) \in k^{n} \backslash\{0\}$.

- the Lie algebra ${\overline{\mathfrak{l}^{\prime}}}_{(b)}(2 n+1, k)$ having the bracket def ined for any $i=1, \ldots, n$ by

$$
\begin{aligned}
& {\left[F_{i}, F_{j}\right]_{b}:=b_{j} F_{i}-b_{i} F_{j}, \quad\left[E_{i}, F_{j}\right]_{b}:=b_{j} E_{i},} \\
& {\left[E_{i}, G\right]_{b}:=\left(2+b_{n}\right) E_{i}, \quad\left[G, F_{i}\right]_{b}:=b_{i}\left(E_{1}+F_{n}\right)-b_{n} F_{i}}
\end{aligned}
$$

for all $b=\left(b_{i}\right) \in k^{n} \backslash\{0\}$.

- the Lie algebra $\overline{\mathfrak{l}}_{(c)}(2 n+1, k)$ having the bracket def ined for any $i=1, \ldots, n$ by

$$
\left[E_{i}, G\right]_{c}:=(1+c) E_{i}, \quad\left[G, F_{i}\right]_{c}:=(1-c) F_{i}
$$

for all $c \in k$.

Thus the factorization index $[\mathfrak{m}(2 n+2, k): k H]^{f}$ is equal to the number of types of isomorphisms of Lie algebras of the set

$$
\left\{\overline{\mathfrak{l}}_{(a)}(2 n+1, k), \overline{\mathfrak{l}}_{(b)}(2 n+1, k),{\overline{\mathfrak{l}^{\prime \prime}}}_{(c)}(2 n+1, k) \mid a, b \in k^{n} \backslash\{0\}, c \in k\right\} .
$$

Proof. As in the proof of Proposition 4.4 we make use of [3, Theorem 4.3]. More precisely, this implies that all complements $\mathfrak{C}$ of $k H$ in $\mathfrak{m}(2 n+2, k)$ are isomorphic to an $r$-deformation of $\mathfrak{l}(2 n+1, k)$, for some deformation map $r: \mathfrak{l}(2 n+1, k) \rightarrow k H$ of the matched pair (4.10). These are described in Lemma 4.7. By a straightforward computation it can be seen that $\overline{\mathfrak{l}}_{(a)}(2 n+1, k)$ is exactly the complement corresponding to the deformation map given by $(4.11), \overline{\mathfrak{l}}_{(b)}(2 n+1, k)$ corresponds to the deformation map given by $(4.12)$ while ${\overline{\mathfrak{l}^{\prime \prime}}}_{(c)}(2 n+1, k)$ is implemented by the deformation map given by (4.13).

Example 4.9. Let $k$ be a field of characteristic $\neq 2$. Then, the factorization index $[\mathfrak{m}(4, k):$ $k H]^{f}$ depends essentially on the field $k$. We will prove that all complements of $k H$ in $\mathfrak{m}(4, k)$ are isomorphic to a Lie algebra of the form:

$$
L_{\alpha}:[x, z]=x, \quad[y, z]=\alpha y, \quad \text { with } \quad \alpha \in k .
$$

Hence, $[\mathfrak{m}(4, k): k H]^{f}=\infty$, if $|k|=\infty$ and $[\mathfrak{m}(4, k): k H]^{f}=\left(1+p^{n}\right) / 2$, if $|k|=p^{n}$, where $p \geq 3$ is a prime number. Indeed, for $n=1$, the Lie algebras described in Proposition 4.8 become

$$
\begin{array}{ll}
\overline{\mathfrak{l}}_{(a)}(3, k): & {[E, F]_{a}:=-a F, \quad[E, G]_{a}:=-a F, \quad[G, F]_{a}:=(2-a) F,} \\
{\overline{\mathfrak{l}^{\prime}}}_{(b)}(3, k): & {[E, F]_{b}:=b E, \quad[E, G]_{b}:=(2+b) E, \quad[G, F]_{b}:=b E,} \\
{\overline{\mathfrak{l}^{\prime \prime}}}_{(c)}(3, k): & {[E, G]_{c}:=(1+c) E, \quad[G, F]_{c}:=(1-c) F}
\end{array}
$$

$a, b \in k^{*}, c \in k$. To start with, we should notice that the first two Lie algebras $\overline{\mathfrak{l}}_{(a)}$ and $\overline{\mathfrak{l}}_{(b)}$ are isomorphic for all $a, b \in k^{*}$. The isomorphism $\gamma: \overline{\mathfrak{l}}_{(a)} \rightarrow \overline{\mathfrak{l}}_{(b)}$ is given as follows

$$
\begin{aligned}
& \gamma(E):=2^{-1}(b-a) E+2^{-1}(b-a+2) F+2^{-1}(a-b) G, \quad \gamma(F):=E, \\
& \gamma(G):=2^{-1}(b-a+4) E+2^{-1}(b-a+4) F+2^{-1}(a-b-2) G .
\end{aligned}
$$


Moreover, the map $\varphi: \overline{\mathfrak{l}}_{(a)} \rightarrow L_{0}$ given by

$$
\varphi(E):=y+a z, \quad \varphi(F):=x, \quad \varphi(G):=x+y+(a-2) z
$$

is an isomorphism of Lie algebras for all $a \in k^{*}$. Therefore, the first two Lie algebras are both isomorphic to $L_{0}$ for all $a, b \in k^{*}$. We are left to study the family ${\overline{\mathfrak{l}^{\prime \prime}}}_{(c)}$. If $c=-1$ then ${\overline{\mathfrak{l}^{\prime \prime}}}_{(-1)}$ is again isomorphic to $L_{0}$. Suppose now that $c \neq-1$. Then the map $\psi: \overline{\mathfrak{l}^{\prime \prime}}{ }_{(c)} \rightarrow L_{(c-1)(c+1)^{-1}}$ given by

$$
\psi(E):=x, \quad \psi(F):=y, \quad \psi(G):=(c+1) z
$$

is an isomorphism of Lie algebras. Finally, we point out here that if $\alpha \notin\left\{\beta, \beta^{-1}\right\}$ then $L_{\alpha}$ is not isomorphic to $L_{\beta}$ (see, for instance [9, Exercise 3.2]) and the conclusion follows.

Remark 4.10. We end this section with two more applications. The deformation of a given Lie algebra $\mathfrak{h}$ associated to a matched pair $(\mathfrak{g}, \mathfrak{h}, \triangleright, \triangleleft)$ of Lie algebras and to a deformation map $r$ as defined by (2.3) is a very general method of constructing new Lie algebras out of a given Lie algebra. It is therefore natural to ask if the properties of a Lie algebra are preserved by this new type of deformation. We will see that in general the answer is negative. First of all we remark that the Lie algebra $\mathfrak{h}:=\mathfrak{l}(2 n+1, k)$ is metabelian, that is $[[\mathfrak{h}, \mathfrak{h}],[\mathfrak{h}, \mathfrak{h}]]=0$. Now, if we look at the matched pair deformation $\mathfrak{h}_{r}=\mathfrak{l}_{(a)}(2 n+1, k)$ of $\mathfrak{h}$ given by (4.9) of Proposition 4.4, for $a=\left(a_{i}\right) \in k^{n} \backslash\{0\}$ we can easily see that $\mathfrak{l}_{(a)}(2 n+1, k)$ is not a metabelian Lie algebra, but a 3-step solvable Lie algebra. Thus the property of being metabelian is not preserved by the $r$-deformation of a Lie algebra.

Next we consider an example of a somewhat different nature. First recall [18] that a Lie algebra $\mathfrak{h}$ is called self-dual (or metric) if there exists a non-degenerate invariant bilinear form $B: \mathfrak{h} \times \mathfrak{h} \rightarrow k$, i.e. $B([a, b], c)=B(a,[b, c])$, for all $a, b, c \in \mathfrak{h}$. Self-dual Lie algebras generalize finite-dimensional complex semisimple Lie algebras (the second Cartan's criterion shows that any finite-dimensional complex semisimple Lie algebra is self-dual since its Killing form is nondegenerate and invariant). Besides the mathematical interest in studying self-dual Lie algebras, they are also important and have been intensively studied in physics $[10,20]$. Now, $\mathfrak{h}:=$ $\mathfrak{l}(2 n+1, k)$ is not a self-dual Lie algebra since if $B: \mathfrak{l}(2 n+1, k) \times \mathfrak{l}(2 n+1, k) \rightarrow k$ is an arbitrary invariant bilinear form then we can easily prove that $B\left(E_{i},-\right)=0$ and thus any invariant form is degenerate. On the other hand, the $r$-deformation of $\mathfrak{l}(2 n+1, k)$ denoted by $\mathfrak{l}_{(0)}^{\prime \prime}(2 n+1, k)$ in Remark 4.5 is self-dual since it is just the $(2 n+1)$-dimensional Abelian Lie algebra.

\section{Two open questions}

The paper is devoted to the factorization problem and its converse, the classifying complements problem, at the level of Lie algebras. Both problems are very difficult ones; even the case considered in this paper, namely $\mathfrak{g}=k_{0}$, illustrates the complexity of the two problems. We end the paper with the following two open questions:

Question 1. Let $n \geq 2$. Does there exist a Lie algebra $\mathfrak{h}$ and a matched pair of Lie algebras $(\mathfrak{g l}(n, k), \mathfrak{h}, \triangleleft, \triangleright)$ such that $\mathfrak{g l}(n, k) \bowtie \mathfrak{h} \cong \mathfrak{g l}(n+1, k)$ ?

A more restricted version of this question is the following: does the canonical inclusion $\mathfrak{g l}(n, k) \hookrightarrow \mathfrak{g l}(n+1, k)$ have a complement that is a Lie subalgebra of $\mathfrak{g l}(n+1, k)$ ? Although it seems unlikely for such a complement to exist we could not find any proof or reference to this problem in the literature.

Secondly, having [2, Corollary 3.2] as a source of inspiration we ask:

Question 2. Let $n \geq 2$. Does there exist a matched pair of Lie algebras $(\mathfrak{g}, \mathfrak{h}, \triangleleft, \triangleright)$ such that any $n$-dimensional Lie algebra $\mathfrak{L}$ is isomorphic to an $r$-deformation of $\mathfrak{h}$ associated to this matched pair? 
At the level of groups, question 2 has a positive answer by considering the canonical matched pair associated to the factorization of $S_{n+1}$ by $S_{n}$ and the cyclic group $C_{n}$.

\section{Acknowledgements}

We would like to thank the referees for their comments and suggestions that substantially improved the first version of this paper. A.L. Agore is research fellow 'Aspirant' of FWOVlaanderen. This work was supported by a grant of the Romanian National Authority for Scientific Research, CNCS-UEFISCDI, grant no. 88/05.10.2011.

\section{References}

[1] Agore A.L., Bontea C.G., Militaru G., Classifying bicrossed products of Hopf algebras, Algebr. Represent. Theory 17 (2014), 227-264, arXiv:1205.6110.

[2] Agore A.L., Militaru G., Classifying complements for groups. Applications, arXiv:1204.1805.

[3] Agore A.L., Militaru G., Classifying complements for Hopf algebras and Lie algebras, J. Algebra 391 (2013), 193-208, arXiv:1205.6564.

[4] Agore A.L., Militaru G., Extending structures for Lie algebras, Monatsh. Math. 174 (2014), 169-193, arXiv:1301.5442.

[5] Andrada A., Barberis M.L., Dotti I.G., Ovando G.P., Product structures on four dimensional solvable Lie algebras, Homology Homotopy Appl. 7 (2005), 9-37, math.RA/0402234.

[6] Andrada A., Salamon S., Complex product structures on Lie algebras, Forum Math. 17 (2005), 261-295, math.DG/0305102.

[7] Benayadi S., Structure of perfect Lie algebras without center and outer derivations, Ann. Fac. Sci. Toulouse Math. 5 (1996), 203-231.

[8] de Graaf W.A., Classification of solvable Lie algebras, Experiment. Math. 14 (2005), 15-25, math.RA/0404071.

[9] Erdmann K., Wildon M.J., Introduction to Lie algebras, Springer Undergraduate Mathematics Series, Springer-Verlag London, Ltd., London, 2006.

[10] Figueroa-O'Farrill J.M., Stanciu S., On the structure of symmetric self-dual Lie algebras, J. Math. Phys. 37 (1996), 4121-4134, hep-th/9506152.

[11] Fisher D.J., Gray R.J., Hydon P.E., Automorphisms of real Lie algebras of dimension five or less, J. Phys. A: Math. Theor. 46 (2013), 225204, 18 pages, arXiv:1303.3376.

[12] Hofmann K.H., Lie algebras with subalgebras of co-dimension one, Illinois J. Math. 9 (1965), 636-643.

[13] Humphreys J.E., Introduction to Lie algebras and representation theory, Graduate Texts in Mathematics, Vol. 9, Springer-Verlag, New York - Berlin, 1972.

[14] Jiang C.P., Meng D.J., Zhang S.Q., Some complete Lie algebras, J. Algebra 186 (1996), 807-817.

[15] Lu J.H., Weinstein A., Poisson Lie groups, dressing transformations, and Bruhat decompositions, J. Differential Geom. 31 (1990), 501-526.

[16] Majid S., Physics for algebraists: noncommutative and noncocommutative Hopf algebras by a bicrossproduct construction, J. Algebra 130 (1990), 17-64.

[17] Majid S., Foundations of quantum group theory, Cambridge University Press, Cambridge, 1995.

[18] Medina A., Revoy P., Algèbres de Lie et produit scalaire invariant, Ann. Sci. École Norm. Sup. 18 (1985), $553-561$.

[19] Michor P.W., Knit products of graded Lie algebras and groups, Rend. Circ. Mat. Palermo (2) Suppl. 22 (1990), 171-175, math.GR/9204220.

[20] Pelc O., A new family of solvable self-dual Lie algebras, J. Math. Phys. 38 (1997), 3832-3840, physics/9709009.

[21] Popovych R.O., Boyko V.M., Nesterenko M.O., Lutfullin M.W., Realizations of real low-dimensional Lie algebras, J. Phys. A: Math. Gen. 36 (2003), 7337-7360, math-ph/0301029.

[22] Su Y., Zhu L., Derivation algebras of centerless perfect Lie algebras are complete, J. Algebra 285 (2005), 508-515, math.QA/0511550. 